\title{
PERMEABILITY ANALYSIS FOR THERMAL BINDER REMOVAL FROM GREEN CERAMIC BODIES
}

\author{
Jeong Woo Yun \\ Dr. Stephen J. Lombardo Dissertation Supervisor
}

\begin{abstract}
The permeability of ceramic green tapes influences the ceramic process steps such as binder removal in which the permeability partially controls the decomposition rate in order to avoid failure of the body, because the permeability relieves the internal pressure build up. The tapes are comprised of barium titanate as the dielectric materials, and poly(vinyl butyral) and dioctyl phthalate as the main binder mixture.
\end{abstract}

The flow though single capillary in the tape was analyzed in terms of models for describing Knudsen, slip, and Poiseuille flow as transport mechanisms. An analytical solution was derived, and compared to an approximate solution. The relative contributions of these three flow mechanisms were then analyzed for different ratios of the mean free path to pore size and for different pressure driving forces.

The permeability of green tapes which are unlaminated and laminated was determined as a function of binder content for binder removed by air oxidation. The flow in porous media through the tapes was analyzed in terms of models for describing Knudsen, slip, and Poiseuille flow mechanisms. With measurement of flow, the characteristic pore size was determined to be $1-2 \mu \mathrm{m}$ for unlaminated and $0.5-1 \mu \mathrm{m}$ for 
laminated samples as a function of binder loading. Poiseuille flow was thus the dominant transport mechanism contributing to the flux in both the unlaminated and laminated samples and therefore Darcy's Law was used to determine the permeability. The number of tapes strongly affects to the permeability for the laminated samples. The permeability was also determined from micro-structural attributes in terms of the specific surface, the pore fraction, and terms of the specific surface, the pore fraction, and terms to account for tortuosity and constrictions.

The permeability and adhesion strength of laminated green ceramic tapes were determined. Both the flow in porous media and the adhesion strength were seen to depend on the lamination conditions of time, temperature, and pressure. The adhesion strength was seen to increase with increasing lamination conditions of time, temperature and pressure, whereas the permeability decreased. 